\title{
ABSOLUTE SUBRETRACTS AND WEAK INJECTIVES IN CONGRUENCE MODULAR VARIETIES
}

\author{
BRIAN A. DAVEY AND L. G. KOVÁCS
}

\begin{abstract}
Absolute subretracts and weak injectives in congruence modular varieties of universal algebras are investigated by focusing attention on the directly indecomposibles. The proofs rely on a congruence modular version of generalized direct products (direct products with amalgamation) and on the generalized Jónsson Lemma for congruence modular varieties. The results have immediate application to varieties of groups or rings.
\end{abstract}

1. Introduction. Weak injectives and absolute subretracts were first considered in a universal-algebraic setting in G. Grätzer and H. Lakser [7] and have since been studied in a range of varieties; see [1] by $\mathrm{H}$. Werner and the first author, particularly $\$ 1$ and the references given there. As shown in [1], these concepts are particularly tractable in congruence distributive varieties. Unfortunately, there appears to be no satisfactory theory for varieties in general.

In particular, there is little directly relevant literature for varieties of groups: apparently none on weak injectives; only negatives on absolute subretracts except the relevant half-page (p. 144) of Hanna Neumann's book [13] (where they are called 'closed') and its amplification in [12] of M. F. Newman and the second author.

Our first aim here is to extend to congruence modular varieties one of the claims published (without the easy proof) for finite groups on p. 144 of [13], and to provide a parallel for weak injectives.

The reason we can go this far, and no further, is that even the statements rely on commutators (of congruences) for which a fully workable theory has been developed only in the congruence modular case. For the relevant definitions the reader is referred forward to the body of the paper. We note here that the terms in our theorems have their usual meaning for groups, but "abelian ring" means zeroring, a finitely subdirectly irreducible ring "has trivial center" means that either the left or the right annihilator of the ring is 0 , while a subdirectly irreducible ring "has nonabelian monolith" means that its unique minimal ideal is not a zeroring.

1.1 TheOREM. Let $A$ be a nonabelian, directly indecomposable algebra in a congruence modular variety $\mathscr{K}$. If $A$ is an absolute subretract in $\mathscr{K}$, then $A$ is finitely subdirectly irreducible and the center of $A$ is trivial. If $A$ is weakly injective in $\mathscr{K}$, then

\footnotetext{
Received by the editors August 29, 1985.

1980 Mathematics Subject Classification (1985 Revision). Primary 08B30, 08B10; Secondary 20E10, 16 A52.

Key words and phrases. Injectivity, congruence modularity, varieties of universal algebras, groups, rings. 
$A$ is prime; in particular, if $A$ is also finite, then it is subdirectly irreducible with nonabelian monolith.

For example, the symmetric group of degree 3 is not weakly injective in its variety, though (as hinted in [13] and mentioned in [12]) it is easily seen to be an absolute subretract there. Theorem 1.1 is proved in $\$ 4$.

The groups proof used generalized direct products (direct products with amalgamation); perhaps the most interesting aspect of the present proof is the use of that construction in the general congruence modular context. We expect that by using generalized subdirect products instead, one could strengthen the conclusion "the center of $A$ is trivial" to "the annihilators of nonabelian congruences of $A$ are all trivial", as was done for groups in some 1964 correspondence between M. F. Newman and the second author. Without being named, such subdirect products with amalgamation have been used in the general congruence modular context by $\mathrm{R}$. S. Freese and R. N. McKenzie; see the proof of Theorem 7 in [4] and the last paragraph of $\S 7$ in [5]. We shall not pursue this point here, partly because even this stronger form of the first half of Theorem 1.1 has no converse: as mentioned in [13], M. F. Newman and the second author had found that a finite subdirectly irreducible group can satisfy the stronger conclusion without being an absolute subretract in the variety it generates. We sketch their example in the final section of this paper.

By contrast, we can give a partial converse to the second half of Theorem 1.1. A variety $\mathscr{K}$ is called subregular if for all $A \in \mathscr{K}$, all $B \leqslant A$ and all $\alpha, \beta \in \operatorname{Con} A$, $[b] \alpha=[b] \beta$ for all $b \in B$ implies $\alpha=\beta$. Varieties of groups and rings are subregular; all subregular varieties are congruence modular (but not vice versa). See [2] by K. R. Miles, V. J. Schumann and the first author.

1.2 TheOREM. If $A$ is finite and subdirectly irreducible with nonabelian monolith, and if $\operatorname{Var} A$ is subregular, then $A$ is weakly injective in $\operatorname{Var} A$.

The principal tool in the proof of Theorem 1.2, to be found in $\S 5$, is the congruence modular generalization of Jónsson's Lemma (see $[3,5,10])$. We also prove there some variants of Theorem 1.2, in which the finiteness assumption on $A$ is relaxed (though not simply dropped) and $\operatorname{Var} A$ is replaced by a larger subregular variety. These results also show us algebras that are weakly injective in a variety $\mathscr{K}$ without necessarily generating it: however, they do not even begin to give us an overview of all weakly injectives in any given $\mathscr{K}$. The only conclusive result we can offer in that direction is the following. For any algebra $A$ and Boolean algebra $B$ the algebra of all continuous maps from the Stone space $X_{B}$ of $B$ into $A$ (with the discrete topology) is called a bounded Boolean power of $A$. (For a brief discussion of functional completeness see $\$ 5$ below.)

1.3 TheOREM. If $\operatorname{Var} A$ is subregular and if $A$ is finite and functionally complete ( for instance, because $\operatorname{Var} A$ is congruence permutable and $A$ nonabelian simple), then the weak injectives of $\operatorname{Var} A$ are precisely the bounded Boolean powers of $A$ by complete Boolean algebras. 
This relies on, and answers a question raised in, B. A. Davey and H. Werner [1]; it applies, in particular, to the case where $A$ is a finite simple nonabelian group or the ring of all $n \times n$ matrices over a finite field.

The results above apply only to nonabelian algebras. For abelian algebras the distinction between weakly injectives and injectives disappears. The following result is proved at the end of $\S 3$.

1.4 THEOREM. If an abelian algebra $A$ is weakly injective in a congruence modular variety $\mathscr{K}$, then it is injective in $\mathscr{K}$.

Theorem 1.4 does not extend to absolute subretracts since it is easy to see that the cyclic group of order 9 is an absolute subretract but not an injectuve, and hence not even a weakly injective, in the variety of groups usually denoted by $\mathscr{A}_{3} \mathscr{A}_{2} \vee \mathscr{A}_{9}$. In this context, it should be noted that in varieties of groups all injectives are known: O. C. Garcia and F. Larrión [6] determine the abelian injectives in each variety of groups, while M. F. Newman and the second author [12] show that the injectives in any variety of groups must be abelian.

We are indebted to Dr. M. F. Newman for his permission to use unpublished material of which he was joint author, and for many stimulating conversations.

2. Preliminaries. We require some definitons concerning injectivity, a generalization of the concept of congruence regularity, and some properties of the commutator in a congruence modular variety.

An algebra $A$ is (weakly) injective relative to a class $\mathscr{K}$ of algebras if every (surjective) homomorphism $\psi: B \rightarrow A$, where $B$ is a subalgebra of some $C \in \mathscr{K}$, can be extended to a homomorphism $\psi^{\prime}: C \rightarrow A$. If $A \in \mathscr{K}$ we say that $A$ is (weakly) injective in $\mathscr{K}$. If $A$ is weakly injective in $\mathscr{K}$, then $A$ is an absolute subretract in $\mathscr{K}$ (also referred to as closed in $\mathscr{K}$ ); that is, if $\psi: A \rightarrow B$ is an embedding with $B \in \mathscr{K}$, then there is a homomorphism $\psi^{\prime}: B \rightarrow A$ satisfying $\psi \circ \psi^{\prime}=\mathrm{id}_{A}$.

The lattice of congruences on $A$ is denoted by Con $A$ with smallest element 0 and largest element 1. For $a \in A$ and $\alpha \in \operatorname{Con} A,[a] \alpha$ denotes the congruence class of $a$ in $\alpha$ and for $B \subseteq A$ we define $[B] \alpha=\bigcup([b] \alpha \mid b \in B)$.

An algebra $A$ has subregular congruences if one of the following equivalent conditions holds (see B. A. Davey, K. R. Miles, and V. J. Schumann [2]).

(i) For all $B \leqslant A$ and all $\alpha, \beta \in \operatorname{Con} A,[b] \alpha=[b] \beta$ for all $b \in B$ implies $\alpha=\beta$;

(ii) For all $B \leqslant A$ and all $\alpha, \beta \in \operatorname{Con} A, \alpha \uparrow B \subseteq \beta \uparrow B$ and $[B] \alpha=B$ implies $\alpha \subseteq \beta$.

If every algebra $A \in \mathscr{K}$ has subregular congruences, then we say that $\mathscr{K}$ is subregular. Both groups and rings have subregular congruences.

For a variety $\mathscr{K}$ congruence subregularity implies congruence modularity (that is, Con $A$ is a modular lattice for all $A \in \mathscr{K}$ ). Whenever $\mathscr{K}$ is congruence modular we may call upon commutator theory, for which J. Hagemann and C. Herrmann [10], C. Hermann [11], H. P. Gumm [8, 9], R. S. Freese and R. N. McKenzie [5], R. Freese [3] and W. Taylor [14] are basic references. We now collect together the 
commutator-theoretic results needed later; proofs can be found in one or more of the references listed above. For the remainder of this section all algebras are assumed to be in a congruence modular variety.

Let $\alpha, \beta \in$ Con $A$ and let $\Delta_{\alpha}^{\beta}$ be the congruence on $\alpha$ (viewed as a subalgebra of $\left.A^{2}\right)$ generated by $\{((a, a),(b, b)) \mid a \beta b\}$; note that $\Delta_{\alpha}^{\beta} \leqslant(\beta \times \beta) \uparrow \alpha$. Then the commutator $[\alpha, \beta] \in \operatorname{Con} A$ is defined by

$$
[\alpha, \beta]=\left\{(a, b) \mid(\exists c \in A)(a, c) \Delta_{\alpha}^{\beta}(b, c)\right\} .
$$

For groups and rings, where we may replace congruences by normal subgroups and two-sided ideals, respectively, the commutator is familiar: it is the usual commutator for normal subgroups while for ideals $I$ and $J$, we have $[I, J]=I J+J I$.

2.1 Proposition. (i) $[\alpha, \beta] \leqslant \alpha \wedge \beta$.

(ii) $[\alpha, \beta]=[\beta, \alpha]$.

(iii) $\left[\alpha, \mathrm{V}\left(\beta_{i} \mid i \in I\right)\right]=\mathrm{V}\left(\left[\alpha, \beta_{i}\right] \mid i \in I\right)$.

(iv) If $\alpha_{1}, \beta_{1} \in \operatorname{Con} A_{1}$ and $\alpha_{2}, \beta_{2} \in \operatorname{Con} A_{2}$, then in $\operatorname{Con} A_{1} \times A_{2}$

$$
\left[\alpha_{1} \times \alpha_{2}, \beta_{1} \times \beta_{2}\right]=\left[\alpha_{1}, \beta_{1}\right] \times\left[\alpha_{2}, \beta_{2}\right] .
$$

(v) If $B \leqslant A$ and $\alpha, \beta \in \operatorname{Con} A$, then $[\alpha \uparrow B, \beta \uparrow B] \leqslant[\alpha, \beta] \uparrow B$.

Commutator theory began in congruence permutable varieties where we have a Mal'cev term (that is, a ternary term $p$ satisfying $p(x, x, y)=p(y, x, x)=y$ ). More generally in congruence modular varieties there is always an almost-Mal'cev term (called a difference term in [5]).

2.2 Proposition. Every congruence modular variety $\mathscr{K}$ has a ternary term $p$ such that

(i) $p(y, x, x)=y$ is an identity in $\mathscr{K}$, and

(ii) for all $\alpha \in \operatorname{Con} A$, xay implies $p(x, x, y)[\alpha, \alpha] y$.

A congruence $\alpha$ is called abelian if $[\alpha, \alpha]=0$. The almost-Mal'cev term of 2.2 yields a beautiful characterization of abelian congruences.

2.3 Proposition. (a) $[\alpha, \alpha]=0$ if and only if

(i) $x \alpha y$ implies $p(x, x, y)=y$, and

(ii) $p: B \rightarrow A$ is a (surjective) homomorphism where

$$
B:=\left\{(a, b, c) \in A^{3} \mid a \alpha b \alpha c\right\} .
$$

(b) If $[\alpha, \alpha]=0$ and $a \alpha b$, then $p(a, b, p(b, a, b))=b$.

(To see (b) use 2.2 and the fact that $p: B \rightarrow A$ is a homomorphism to calculate $p(p(a, b, b), p(a, a, b), p(b, a, b))$ in two different ways.)

An algebra $A$ is called abelian if $[1,1]=0$ in Con $A$. Note from 2.2 that an almost-Mal'cev term becomes a Mal'cev term on an abelian algebra.

2.4 Proposition. $A$ is abelian if and only if there are congruences $\alpha, \beta, \gamma$ on $A^{2}$ such that $\{0, \alpha, \beta, \gamma, 1\}$ is a sublattice of $\operatorname{Con} A^{2}$ isomorphic to the five-element, modular, nondistributive lattice $M_{3}$. 
We shall need the Hagemann-Herrmann-Freese-McKenzie-Hrushovskii generalization of Jónsson's Lemma. The largest congruence $\beta$ satisfying $[\alpha, \beta]=0$ is called the annihilator (or centralizer) of $\alpha$ and is denoted by ann $\alpha$. Recall that $A$ is subdirectly irreducible if 0 is completely meet-irreducible in $\operatorname{Con} A$; then the unique cover of 0 in Con $A$ is called the monolith of $A$ and is denoted by $\mu$.

2.5 The Generalized Jónsson Lemma. Let $\left(C_{i} \mid i \in I\right)$ be a family of algebras from a congruence modular variety, let $A$ be subdirectly irreducible with monolith $\mu$, let $B$ be a subalgebra of $\Pi\left(C_{i} \mid i \in I\right)$ and let $\phi: B \rightarrow A$ be a surjective homomorphism. Then there exists an ultrafilter $F$ on $I$ such that $\theta_{F} \mid B \leqslant \operatorname{ker}(\phi \circ n)$ where $n$ : $A \rightarrow A /$ ann $\mu$ is the natural map. Hence there is a homomorphism $\psi: B /\left(\theta_{F} \mid B\right) \rightarrow$ $A$ /ann $\mu$ such that the diagram below commutes.

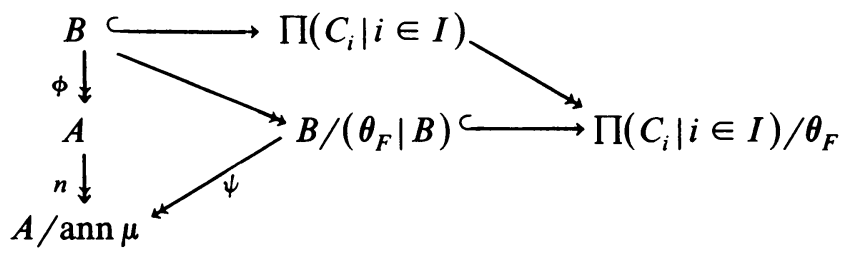

In terms of the usual class operators, we have $A / \operatorname{ann} \mu \in \mathbf{H S P}_{u}(\mathscr{A})$ where $\mathscr{A}=\left\{C_{i} \mid i\right.$ $\in I\}$.

Finally, the following easy application of the modularity of Con $A \times B$ will find several applications.

2.6 LemMA. Every congruence on $A \times B$ below $\alpha \times 0$ is of the form $\alpha^{\prime} \times 0$ for some $\alpha^{\prime} \leqslant \alpha$.

3. Central cubes. We need some further preliminaries on central congruences, that is, congruences $\alpha \in \operatorname{Con} A$ satisfying $[\alpha, 1]=0$. The largest central congruence, ann 1 , is called the center of $A$ and is denoted by $\zeta_{A}$ or simply $\zeta$. Throughout this section we work in an arbitrary but fixed congruence modular variety with almost$\mathrm{Mal}^{\prime} \mathrm{cev}$ term $p$. Our approach relies heavily on the results of $\$ 9$ of [9] by H. P. Gumm which we summarize in the paragraph below.

Let $a \in A$ and $\alpha \in \operatorname{Con} A$. For each operation $f$ on $A$ we define $f^{\nabla}$ on $[a] \alpha$ by

$$
f^{\nabla}\left(x_{1}, \ldots, x_{n}\right)=p\left(a, f(a, \ldots, a), f\left(x_{1}, \ldots, x_{n}\right)\right) \text { for } x_{1}, \ldots, x_{n} \in[a] \alpha .
$$

The resulting algebra with base set $[a] \alpha$, which is denoted by $A^{\nabla}[\alpha]_{a}$, is of the same type as $A$ and has a one-element subalgebra $\{a\}$ but need not be in the same variety as $A$. Note that if $\alpha$ is abelian and $\{a\}$ is a one-element subalgebra of $A$, then $A^{\nabla}[\alpha]_{a}$ is just the subalgebra $[a] \alpha$ of $A$. If $\alpha$ is a central congruence, then $A^{\nabla}[\alpha]_{a} \cong A^{\nabla}[\alpha]_{b}$ for all $a, b \in A$; in this case we choose an arbitrary element 0 in $A$ and abbreviate $A^{\nabla}[\alpha]_{0}$ to $A^{\nabla}[\alpha]$. The algebras $\alpha, A^{\nabla}[\alpha]$ and $A$ are closely related.

3.1 Proposition [9]. Let $\alpha$ be a central congruence on $A$.

(i) $A^{\nabla}[\alpha]$ is an abelian algebra.

(ii) $A^{\nabla}[\alpha] \cong \alpha / \Delta_{\alpha}^{1}$.

(iii) $\alpha \cong A \times A^{\nabla}[\alpha]$ : namely, the map $(a, b) \mapsto p(0, a, b)$ is a homomorphism of $\alpha$ onto $A^{\nabla}[\alpha]$ with kernel $\Delta_{\alpha}^{1}$, and an isomorphism from $\alpha$ to $A \times A^{\nabla}[\alpha]$ may be defined by $(a, b) \mapsto(a, p(0, a, b))$, with inverse given by $(a, c) \mapsto(a, p(a, 0, c))$. 
The crucial fact that we need is the isomorphism between the lattice of central congruences on $A$ and the lattice of all subalgebras of $A^{\nabla}[\zeta]$ which contain 0 , mirroring the groups case where every subgroup of the centre is normal. For a subalgebra $S$ of $A^{\nabla}[\zeta]$ containing 0 , define

$$
\Psi(S)=\{(x, y) \in \zeta \mid p(0, x, y) \in S\}
$$

and for a central congruence $\alpha$ define $U(\alpha)=A^{\nabla}[\alpha]$. If $L$ is a lattice and $a \in L$, then the principal ideal $\{x \in L \mid x \leqslant a\}$ is denoted by $\downarrow a$ and its dual by $\uparrow a$.

3.2 PROPOSITION [9]. $U$ and $\Psi$ are mutually inverse lattice isomorphisms between the interval $\downarrow \zeta$ in $\operatorname{Con} A$ and $\operatorname{Sub}_{0} A^{\nabla}[\zeta]$, the lattice of subalgebras of $A^{\nabla}[\zeta]$ which contain 0 .

The following is an easy consequence.

3.3 COROLlARY. Central congruences are regular; that is, if $\alpha$ and $\beta$ are central and have a block in common then $\alpha=\beta$, and, more generally, if $[a] \alpha \subseteq[a] \beta$ for some $a \in A$, then $\alpha \leqslant \beta$.

We are now able to study the passage of central congruences under restriction to a subalgebra. Note that by $2.1(\mathrm{v})$, if $\alpha$ is a central congruence on $A$ and $B \leqslant A$, then $\alpha \uparrow B$ is also central.

3.4 Proposition. Let $A$ be an algebra with subalgebra $B$ containing an element 0 and define

$$
\zeta_{A, B}=\left\{(x, y) \in \zeta_{A} \mid p(0, x, y) \in B\right\} .
$$

(i) $\zeta_{A, B}$ is a central congruence on $A$.

(ii) Restriction is an isomorphism between the intervals $\downarrow\left(\zeta_{A, B}\right)$ in $\operatorname{Con} A$ and $\downarrow\left(\zeta_{A} \uparrow B\right)$ in $\operatorname{Con} B$.

(iii) If $\beta \leqslant \zeta_{A} \uparrow B$, then the unique congruence $\bar{\beta}$ on $A$ such that $\bar{\beta} \leqslant \zeta_{A, B}$ and $\bar{\beta} \uparrow B=\beta$ is given by

$$
\bar{\beta}=\left\{(x, y) \in \zeta_{A} \mid p(0, x, y) \in[0] \beta\right\} .
$$

(iv) If $\alpha$ is a central congruence on $A$ and $B$ is a union of $\alpha$-blocks (that is, $[B] \alpha=B)$, then restriction is an isomorphism between the intervals $\downarrow \alpha$ in Con $A$ and $\downarrow(\alpha \uparrow B)$ in $\operatorname{Con} B$.

Proof. It follows from 3.2 that if $S \in \operatorname{Sub}_{0} A^{\nabla}[\zeta]$, then $U$ and $\Psi$ form an inverse pair of lattice isomorphisms between $\downarrow \Psi(S)$ and $\operatorname{Sub}_{0}(S)$. Given $A$ and $B$, choose 0 in $B$ so it can serve both $A$ and $B$, let $\Psi_{A}, U_{A}$ stand for the $\Psi, U$ defined above, and $\Psi_{B}, U_{B}$ for the maps similarly defined with $B$ in place of $A$. By 2.1(v), $\zeta_{A} \uparrow B \leqslant \zeta_{B}$. Note that the base set $[0] \zeta_{A} \cap B$ of $U_{B}\left(\zeta_{A} \uparrow B\right)$ is also the intersection of the base sets of $U_{A}\left(\zeta_{A}\right)$ and $U_{B}\left(\zeta_{B}\right)$, and that the operations of these algebras agree on that intersection, so $U_{B}\left(\zeta_{A} \uparrow B\right) \in \operatorname{Sub}_{0} A^{\nabla}\left[\zeta_{A}\right] \cap \operatorname{Sub}_{0} B^{\nabla}\left[\zeta_{B}\right]$. The definitions of $\Psi_{A}$ and $U_{B}$ give that $\Psi_{A}\left(U_{B}\left(\zeta_{A} \uparrow B\right)\right)=\zeta_{A, B}$ : by 3.2, this proves (i). Two applications of the consequence of 3.2 noted at the beginning of this proof yield that the composite $\Psi_{A} U_{B}$ maps $\downarrow\left(\zeta_{A} \uparrow B\right)$ via $\operatorname{Sub}_{0} U_{B}\left(\zeta_{A} \uparrow B\right)$ lattice-isomorphically onto $\downarrow\left(\zeta_{A, B}\right)$. A set-theoretical one-sided inverse of an isomorphism is always the inverse 
isomorphism: hence for (ii) it suffices to show that if $\beta \in \downarrow(\zeta \uparrow B)$ and $\bar{\beta}=$ $\Psi_{A}\left(U_{B}(\beta)\right)$, then $\bar{\beta} \mid B=\beta$. By 3.3 , this will in turn follow if we can show that $[0](\bar{\beta} \uparrow B)=[0] \beta$. Indeed, as $(0, b) \in \zeta_{B}$ implies that $p(0,0, b)=b$ by 2.3(a)(i),

$$
\begin{aligned}
{[0](\bar{\beta} \uparrow B) } & =[0] \bar{\beta} \cap B=\left\{b \in B \mid(0, b) \in \zeta_{A} \& p(0,0, b) \in[0] \beta\right\} \\
& =B \cap[0] \zeta_{A} \cap[0] \beta \\
& =[0] \beta .
\end{aligned}
$$

This proves (ii), and (iii) then follows from the definition of $\Psi_{A} U_{B}$. [One may note that while $\Psi_{A}$ and $U_{B}$ do depend (however harmlessly) on the choice of 0 , their composite (where it is defined) does not.] To deduce (iv), all one needs is that $\alpha \leqslant \zeta_{A}$ and $[B] \alpha=B$ imply $\alpha \leqslant \zeta_{A, B}$; by 3.3, $\alpha \leqslant \zeta_{A, B}$ is equivalent to [0] $\alpha \subseteq[0] \zeta_{A, B}$; and the latter inclusion now holds because by 2.3(a)(i) and the definition of $\zeta_{A, B}$ we have $[0] \zeta_{A, B}=[0] \zeta_{A} \cap B$.

We now apply 3.4 with $A^{3}$ in place of $A$ and $B$ chosen to be $\{(a, b, c) \in$ $\left.A^{3} \mid a \zeta b \zeta c\right\}$. Since the centre of $A^{3}$ is $\zeta^{3}$ and $[B] \zeta^{3}=B$, the restriction map is an isomorphism between the intervals $\downarrow\left(\zeta^{3}\right)$ in Con $A^{3}$ and $\downarrow\left(\zeta^{3} \uparrow B\right)$ in Con $B$.

By 2.3, $p: B \rightarrow A$ is a surjective homomorphism. Denote its kernel by $\pi$, then $B / \pi \cong A$. It is easily seen that $\pi \leqslant \zeta^{3} \mid B$. (Indeed, $(b, c) \in \zeta$ implies that $(p(a, b, c), a)=p((a, a),(b, c),(c, c)) \in \zeta$. It follows that if $p$ agrees on two elements of $B$, then their first coordinates are congruent modulo $\zeta$; as they are elements of $B$ all three coordinates of each are congruent modulo $\zeta$.) Let $\bar{\pi}$ be the unique central congruence on $A^{3}$ satisfying $\bar{\pi} \uparrow B=\pi$; if required, 3.4(iii) can be used to give an explicit description of $\bar{\pi}$.

We regard $A$ as embedded in $A^{3} / \bar{\pi}$ via the isomorphism $A \cong B / \pi$ obtained from $p$. Since $[B] \zeta^{3}=B$ and $\bar{\pi} \leqslant \zeta^{3}$ we have $[B] \bar{\pi}=B$; hence the embedding of $A \cong B / \pi$ into $A^{3} / \bar{\pi}$ is surjective if and only if $A^{3}=B$ if and only if $\zeta=1$ (that is, $A$ is abelian). Trivially we have $\zeta=0$ if and only if $A^{3} / \bar{\pi}=A^{3}$. Thus we get something 'new' precisely when $0<\zeta<1$. We call $A^{3} / \bar{\pi}$ a central cube of $A$.

We note that while $p$ depends on the variety we choose to work in and is not even determined by that, the homomorphism from $B$ to $A$ given by $p$, and so its kernel $\pi$, is independent of these choices. We shall neither need nor prove this here. A construction we would call a central square of $A$, namely $A^{2} / \Delta_{1}^{\zeta}$, occurs in Proposition 7.6 of [5], but without a one-element subalgebra in $A$ there seems to be no general way to embed $A$ into this central square.

Recall that an element $a$ of a lattice with zero is called dense if 0 is the only element $x$ satisfying $a \wedge x=0$. An algebra $A$ is said to be an essential extension of a subalgebra $B$ if $0<\alpha$ in Con $A$ implies $0<\alpha \uparrow B$ in Con $B$.

3.5 THEOREM. If $\zeta$ is dense in Con $A$, then the central cube of $A$ defined above is an essential extension of $A$.

Proof. We must prove that if $\bar{\pi}<\alpha$ in Con $A^{3}$, then $\pi<\alpha \uparrow B$ in Con $B$. Since restriction yields an isomorphism between $\downarrow\left(\zeta^{3}\right)$ and $\downarrow\left(\zeta^{3} \uparrow B\right)$ we have

$$
\bar{\pi}<\alpha \leqslant \zeta^{3} \Rightarrow \pi<\alpha \uparrow B
$$


consequently it suffices to show that if $\zeta$ is dense in $A$, then $\zeta^{3}$ is dense in the interval $\left\{\sigma \in \operatorname{Con} A^{3} \mid \bar{\pi} \leqslant \sigma \leqslant 1\right\}$.

Let $\zeta$ be dense in Con $A$. It is sufficient to prove that if $\alpha \wedge \zeta^{3}=\bar{\pi}$ in Con $A^{3}$, then $\alpha \leqslant \zeta^{3}$. As $\zeta^{3}$ is the centre of $A^{3}$, the additive property of the commutator (2.1(iii)) yields this conclusion if we can prove that $\alpha$ annihilates each of $1 \times 0 \times 0$, $0 \times 1 \times 0$ and $0 \times 0 \times 1$. Denote these congruences by $\iota_{1}, \iota_{2}, \iota_{3}$, respectively. We prove $\left[\alpha, \iota_{2}\right]=0$; the proof of the other two claims are strict analogues of (proper parts of) this proof. By 2.6 every congruence below $\iota_{2}$ is of the form $0 \times \beta \times 0$ for some $\beta \in \operatorname{Con} A$, in particular, $\alpha \wedge \iota_{2}=0 \times \beta \times 0$ for some $\beta \in \operatorname{Con} A$. Note that

$$
0 \times(\beta \wedge \zeta) \times 0 \leqslant \alpha \wedge \zeta^{3}=\bar{\pi} .
$$

Take any element $(a, b)$ of $\beta \wedge \zeta$ and set $\underline{a}=(b, a, b)$ and $\underline{b}=(b, b, b)$. Then

$$
\underline{a} \in B \cap[\underline{b}](0 \times(\beta \wedge \zeta) \times 0) \subseteq B \cap[\underline{b}] \bar{\pi}=[\underline{b}] \pi,
$$

that is, $p(\underline{a})=p(\underline{b})=b$ and so $p(a, b, p(\underline{a}))=a$. On the other hand, $p(a, b, p(\underline{a}))=b$ by $2.3(\mathrm{~b})$. We have proved that $(a, b) \in \beta \wedge \zeta$ implies $a=b$ : so $\beta \wedge \zeta=0$. As $\zeta$ is dense, $\beta=0$ so $\alpha \wedge \iota_{2}=0$. This proves $\left[\alpha, \iota_{2}\right]=0$.

We close this section with a proof of Theorem 1.4, a further application of the abelian algebras $A^{\nabla}[\alpha]$.

Proof of TheOrem 1.4. Let $A$ be an abelian algebra that is weakly injective in a congruence modular variety $\mathscr{K}$. Let $C \geqslant B \stackrel{\phi}{\rightarrow} A$ be given in $\mathscr{K}$ : we have to find an extension $C \rightarrow A$ of $\phi$. Abbreviate $A^{\nabla}[1]$ to $A^{\nabla}$ and consider the composite map $\psi$ :

$$
B \times A^{\nabla} \stackrel{\phi \times \text { id }}{\rightarrow} A \times A^{\nabla} \stackrel{f^{-1}}{\rightarrow} A \times A \rightarrow A,
$$

where $f: A \times A \rightarrow A \times A^{\nabla}$ is the isomorphism given in 3.1(iii) and the last map is the second projection. This $\psi$ is surjective: if $a$ is any element of $A$, take any $b$ in $B$; as

$$
(b, p(0, b \phi, a)) \stackrel{\phi \times \mathrm{id}}{\mapsto}(b \phi, p(0, b \phi, a)) \stackrel{f}{\leftrightarrow}(b \phi, a)
$$

we see that $\psi$ maps $(b, p(0, b \phi, a))$ to $a$. Since $A$ is weakly injective, $\psi$ extends to a homomorphism $\theta: C \times A^{\nabla} \rightarrow A$. The obvious map $c \mapsto(c, 0)$ from $C$ to $C \times A^{\nabla}$ composed with $\theta$ will do as the required extension of $\phi$ : indeed, $(b, 0) \theta=(b, 0) \psi=$ $b \phi$ because

$$
(b, 0) \stackrel{\phi \times \mathrm{id}}{\mapsto}(b \phi, 0) \stackrel{f}{\leftarrow}(b \phi, b \phi)
$$

4. Directly indecomposables. Our aim in this section is to prove Theorem 1.1. A congruence modular variety is assumed throughout.

4.1 LemMA. If $L$ is a modular lattice with zero, if $a \in L$ and $b \in \uparrow a$, and if $b$ is dense in $\uparrow a$, then $b$ is dense in $L$.

Proof. Assume $b$ is dense in $\uparrow a$ and let $x \in L$ with $b \wedge x=0$. Since $b \geqslant a$ we have $b \wedge(x \vee a)=(b \wedge x) \vee a=a$ by modularity. Hence $x \vee a=a$ and thus $x \leqslant a \leqslant b$. Consequently, $x=b \wedge x=0$. 
An algebra $A$ is called finitely subdirectly irreducible if 0 is meet-irreducible in Con $\boldsymbol{A}$.

4.2 TheOREM. Assume that $A$ is a directly indecomposable absolute subretract in $\operatorname{Var} A$. Then $A$ is finitely subdirectly irreducible.

Proof. Assume that $A$ is an absolute subretract in $\operatorname{Var} A$ and is not finitely subdirectly irreducible: we shall find a direct decomposition of $A$. Since 0 is meet-reducible in Con $A$, there exist $\gamma, \delta \in \operatorname{Con} A$ with $\gamma \neq 0, \delta \neq 0$ and $\gamma \wedge \delta=0$. Since Con $A$ is algebraic, meet distributes over arbitrary directed joins and hence we can assume that each of $\gamma$ and $\delta$ is maximal with respect to $\gamma \wedge \delta=0$. Let $\varepsilon$ : $A \rightarrow A / \gamma \times A / \delta$ be the natural embedding. We claim that $A / \gamma \times A / \delta$ is an essential extension of $A \varepsilon$. For notational convenience we identify $A$ with $A \varepsilon$ and so identify $a$ with $([a] \gamma,[a] \delta)$.

Let $\alpha=(\gamma \vee \delta) / \gamma \in \operatorname{Con} A / \gamma$ and $\beta=(\gamma \vee \delta) / \delta \in$ Con $A / \delta$. We shall prove:

(i) $0<\theta \leqslant \alpha \times \beta$ in $\operatorname{Con}(A / \gamma \times A / \delta)$ implies $\theta \uparrow A \neq 0$;

(ii) $\alpha \times \beta$ is dense in $\operatorname{Con}(A / \gamma \times A / \delta)$.

Clearly (i) and (ii) imply that $\varepsilon$ is an essential extension.

In proving (i) we need:

(iii) Restriction to $A$ maps $(\downarrow \alpha) \times\{0\}$ in $\operatorname{Con}(A / \gamma \times A / \delta)$ isomorphically onto $\downarrow \delta$ in $\operatorname{Con} A$;

(iv) Let $\alpha_{0}=\alpha \wedge \zeta_{A / \gamma}$ and $\beta_{0}=\beta \wedge \zeta_{A / \delta}$; then $[A]\left(\alpha_{0} \times \beta_{0}\right)=A$.

Proof of (iii). Consider the composite of the following chain of natural isomorphisms:

$$
\begin{aligned}
\downarrow \delta & \cong\{\sigma \in \operatorname{Con} A \mid \gamma \leqslant \sigma \leqslant \gamma \vee \delta\} & & \text { in Con } A \text { by modularity } \\
& \cong \downarrow \alpha & & \text { in } \operatorname{Con} A / \gamma \\
& \cong(\downarrow \alpha) \times\{0\} & & \text { in } \operatorname{Con}(A / \gamma \times A / \delta) .
\end{aligned}
$$

Under this isomorphism an element $\delta^{\prime}$ of $\downarrow \delta$ maps to $\alpha^{\prime} \times 0$ where $\alpha^{\prime}=\left(\gamma \vee \delta^{\prime}\right) / \gamma$. It remains to verify that $\delta^{\prime}$ is the restriction to $A$ by $\alpha^{\prime} \times 0$ :

$$
\begin{aligned}
([a] \gamma,[a] \delta) \alpha^{\prime} \times 0([b] \gamma,[b] \delta) & \Leftrightarrow a\left(\gamma \vee \delta^{\prime}\right) b \text { and } a \delta b \\
& \Leftrightarrow a \delta \wedge\left(\gamma \vee \delta^{\prime}\right) b \\
& \Leftrightarrow a \delta^{\prime} b \quad \text { by modularity. }
\end{aligned}
$$

Proof of (iv). By (iii), $\alpha_{0}=\left(\gamma \vee \delta_{0}\right) / \gamma$ where $\delta_{0}=\left(\alpha_{0} \times 0\right) \uparrow A$ with $\delta_{0} \leqslant \delta$. Restrictions of central congruences are central (via 2.1(v)) and abelian congruences permute with all congruences (see $[9,5,4]$ ): so $\gamma \vee \delta_{0}=\gamma \circ \delta_{0}$. Similarly, $\beta_{0}=$ $\left(\gamma_{0} \vee \delta\right) / \delta, \gamma_{0}=\left(0 \times \beta_{0}\right) \uparrow A, \gamma_{0} \vee \delta=\gamma_{0} \circ \delta$ and, of course, $\gamma_{0} \circ \delta_{0}=\delta_{0} \circ \gamma_{0}$. Thus

$$
\begin{aligned}
\left(\left[a^{\prime}\right] \gamma,\left[a^{\prime \prime}\right] \delta\right) \alpha_{0} \times \beta_{0}([a] \gamma,[a] \delta) & \in A \Rightarrow a^{\prime}\left(\gamma \circ \delta_{0}\right) a\left(\gamma_{0} \circ \delta\right) a^{\prime \prime} \\
\Rightarrow a^{\prime}\left(\gamma \circ \delta_{0} \circ \gamma_{0} \circ \delta\right) a^{\prime \prime} & \Rightarrow a^{\prime}\left(\gamma \circ \gamma_{0} \circ \delta_{0} \circ \delta\right) a^{\prime \prime} \Rightarrow a^{\prime}(\gamma \circ \delta) a^{\prime \prime} .
\end{aligned}
$$

Hence there exists $a^{*} \in A$ with $\left(\left[a^{\prime}\right] \gamma,\left[a^{\prime \prime}\right] \delta\right)=\left(\left[a^{*}\right] \gamma,\left[a^{*}\right] \delta\right) \in A$.

Proof of (i). Let $0<\theta \leqslant \alpha \times \beta$ with $\theta \uparrow A=0$. As $\theta \wedge(1 \times 0) \leqslant \alpha \times 0$, by 2.6 there is an $\alpha^{\prime}$ in $\downarrow \alpha$ such that $\theta \wedge(1 \times 0)_{\iota}=\alpha^{\prime} \times 0$. As $(\theta \wedge(1 \times 0)) \uparrow A \leqslant \theta \uparrow A$ $=0$, (iii) yields $\alpha^{\prime}=0$; so $\theta \wedge(1 \times 0)=0$ whence $[\theta, 1 \times 0]=0$. Similarly, 
$[\theta, 0 \times 1]=0$; hence $[\theta, 1 \times 1]=0$ and so $\theta$ is central. Thus $\theta \leqslant \zeta_{A / \gamma} \times \zeta_{A / \delta}$ and so

$$
\theta \leqslant(\alpha \times \beta) \wedge\left(\zeta_{A / \gamma} \times \zeta_{A / \delta}\right)=\left(\alpha \wedge \zeta_{A / \gamma}\right) \times\left(\beta \wedge \zeta_{A / \delta}\right)=\alpha_{0} \times \beta_{0} .
$$

Since $[A]\left(\alpha_{0} \times \beta_{0}\right)=A$, by (iv), $\theta \leqslant \alpha_{0} \times \beta_{0}$ implies that $[A] \theta=A$. Thus by 3.4(iv) restriction is an isomorphism between $\downarrow \theta$ in $\operatorname{Con}(A / \gamma \times A / \delta)$ and $\downarrow(\theta \uparrow A)$ in Con $A$. Since $\theta$ ^ $A=0$ we therefore conclude $\theta=0$.

Proof of (ii). Since $\alpha \times \beta=(\alpha \times 1) \wedge(1 \times \beta)$ it suffices to show that $\alpha \times 1$ and $1 \times \beta$ are dense in $\operatorname{Con}(A / \gamma \times A / \delta)$.

First note that $\gamma \vee \delta$ is dense in $\uparrow \gamma \subseteq$ Con $A$. (If $\phi \in \uparrow \gamma$ with $\phi \wedge(\gamma \vee \delta)=\gamma$, then

$$
\phi \wedge \delta=\phi \wedge(\gamma \vee \delta) \wedge \delta=\gamma \wedge \delta=0
$$

yielding $\phi=\gamma$ by the maximality of $\gamma$ with respect to $\gamma \wedge \delta=0$.) Since $\uparrow(0 \times 1)$ in $\operatorname{Con}(A / \gamma \times A / \delta)$ is isomorphic to $\uparrow \gamma$ in Con $A$, it follows that $\alpha \times 1=(\gamma \vee \delta) / \gamma$ $\times 1$ is dense in $\uparrow(0 \times 1) \subseteq \operatorname{Con}(A / \gamma \times A / \delta)$. By symmetry, $1 \times \beta$ is dense in $\uparrow(1 \times 0) \subseteq \operatorname{Con}(A / \gamma \times A / \delta)$. That $\alpha \times 1$ and $1 \times \beta$ are both dense in $\operatorname{Con}(A / \gamma \times A / \delta)$ now follows at once from Lemma 4.1 above.

Hence $A / \gamma \times A / \delta$ is a proper essential extension of $A$. Since an absolute subretract in a variety cannot have an essential extension in that variety, it follows that $\varepsilon$ is an isomorphism and thus $A$ is directly decomposable.

To complete the proof of the first half of Theorem 1.1 suppose that $A$ is a nonabelian, directly indecomposable absolute subretract in $\mathscr{K}$ with a nontrivial center. Since (by 4.2) $A$ is finitely subdirectly irreducible, every nonzero congruence is dense; in particular, $\zeta_{A}$ is dense in Con $A$. Thus by Theorem 3.5, a central cube of $A$ is a proper essential extension of $A$ in $\operatorname{Var} A$, which is impossible because $A$ is an absolute subretract in $\operatorname{Var} A$. Hence $A$ has trivial centre.

We turn now to the second half of Theorem 1.1. An algebra $A$ is prime if it is finitely subdirectly irreducible and every nontrivial congruence on $A$ is nonabelian (see [10]). Consequently, a subdirectly irreducible algebra is prime if and only if its monolith is nonabelian; in particular, a finite algebra is prime precisely when it is subdirectly irreducible with nonablelian monolith.

Let $A$ be nonabelian, directly indecomposable and weakly injective in $\mathscr{K}$. Since $A$ is an absolute subretract in $\mathscr{K}$, it is finitely subdirectly irreducible and has trivial center (by the first half of Theorem 1.1) and to prove that $A$ is prime it remains to show that every nontrivial congruence on $A$ is nonabelian. Suppose that $\alpha \neq 0$ is abelian. Then by 2.3(a), $p$ maps $B=\left\{(a, b, c) \in A^{3} \mid a \alpha b \alpha c\right\}$ homomorphically onto $A$; as $A$ is weakly injective in $\operatorname{Var} A, p$ must be the restriction of a homomorphism $q: A^{3} \rightarrow A$.

By 2.6, $(\operatorname{ker} q) \wedge(0 \times 1 \times 0)=0 \times \alpha^{\prime} \times 0$ for some $\alpha^{\prime} \in$ Con $A$. Let $a\left(\alpha \wedge \alpha^{\prime}\right) b$; then $\underline{a}=(b, a, b)$ and $\underline{b}=(b, b, b)$ both lie in $B$ and are congruent modulo ker $q$, whence $p(\underline{a})=p(\underline{b})=b$. Thus, as in the proof of 3.5, we have

$$
\begin{aligned}
a & =p(a, b, b)=p(a, b, p(\underline{a})) & & \text { as } p(\underline{a})=b \\
& =b & & \text { by } 2.3(\mathrm{~b}) .
\end{aligned}
$$


This proves $\alpha \wedge \alpha^{\prime}=0$, so $\alpha^{\prime}=0$ as $\alpha \neq 0$ and $A$ is finitely subdirectly irreducible; hence $(\operatorname{ker} g) \wedge(0 \times 1 \times 0)=0$. By similar, but simpler, arguments we also obtain $(\operatorname{ker} q) \wedge(1 \times 0 \times 0)=0$ and $(\operatorname{ker} q) \wedge(0 \times 0 \times 1)=0$. Consequently, $\operatorname{ker} q$ avoids and so annihilates the kernel of each coordinate projection on $A^{3}$ : so it annihilates their join and so is central. But $\zeta_{A^{3}}=\left(\zeta_{A}\right)^{3}=0$, therefore, $\operatorname{ker} q=0$; in particular, $p$ is one-to-one. Thus $a \alpha b$ implies $(b, a, a),(a, a, b) \in B$ with $p(b, a, a)=b=$ $p(a, a, b)$ giving $a=b$, contrary to the assumption that $\alpha \neq 0$. Thus, $\alpha$ is nonabelian and this concludes the proof of Theorem 1.1.

5. Subdirectly irreducibles with nonabelian monolith. We now address the question of when a subdirectly irreducible algebra with nonabelian monolith is weakly injective in a variety containing it. Along the way we prove Theorem 1.2.

Throughout this section we shall be working with the variety $\operatorname{Var} \mathscr{A}$ generated by some class $\mathscr{A}$ of algebras. Just as in the congruence distributive case, the Generalized Jónsson Lemma greatly restricts the whereabouts of a subdirectly irreducible algebra with nonabelian monolith; we omit the proof.

5.1 Lemma. Assume that $\operatorname{Var} \mathscr{A}$ is congruence modular and let $A \in \operatorname{Var} \mathscr{A}$ be subdirectly irreducible with nonabelian monolith. Then $A \in \mathbf{H S P}_{u}(\mathscr{A})$ so if $\mathscr{A}$ is a finite set of finite algebras, then $A \in \mathscr{H} \mathscr{S}(\mathscr{A})$ and hence $A$ is finite. If $A$ is also weakly injective in $\operatorname{Var} \mathscr{A}$, then $A \in \mathbf{H P}_{U}(\mathscr{A})$ with $A \in \mathbf{H}(\mathscr{A})$ when $\mathscr{A}$ is a finite set of finite algebras.

Most results in this section assume congruence modularity only because they need 5.1 (or the Generalized Jónsson Lemma itself). Similarly, the stronger assumption of subregularity is imposed only to make the following lemma available.

5.2 Lemma. Assume that $\mathbf{S}(\mathscr{A})$ is subregular. If an algebra $A$ is (weakly) injective relative to $\mathscr{A}$, then $A$ is (weakly) injective relative to $\mathrm{HS}(\mathscr{A})$.

Proof. As $A$ is (weakly) injective relative to $\mathscr{A}$, it is obviously (weakly) injective relative to $\mathbf{S}(\mathscr{A})$. Let $B \leqslant C \in \mathbf{H S}(\mathscr{A})$ and let $\psi: B \rightarrow A$ be the map to be extended to $C$. Write $C$ as $D \phi$ for some $D \in \mathbf{S}(\mathscr{A})$ and some $\phi: D \rightarrow C$.

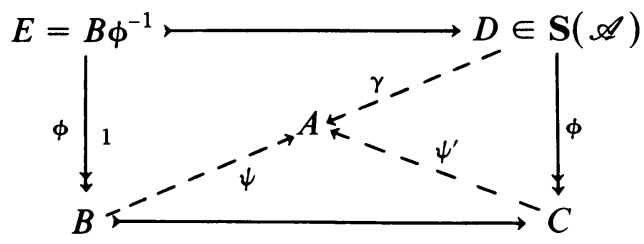

Let $\phi_{1}=\phi \uparrow E$ where $E=B \phi^{-1}$. Since $A$ is (weakly) injective relative to $\mathbf{S}(\mathscr{A})$, there is a homomorphism $\gamma: D \rightarrow A$ making the quadrangle $A D E B$ commute. It is easily seen that $(\operatorname{ker} \phi) \uparrow E \subseteq(\operatorname{ker} \gamma) \uparrow E$ and since $[E] \operatorname{ker} \phi=E$, the subregularity of $D \in \mathbf{S}(\mathscr{A})$ gives $\operatorname{ker} \phi \subseteq \operatorname{ker} \gamma$. Thus there exists a homomorphism $\psi^{\prime}: C \rightarrow A$ such that $\phi \circ \psi^{\prime}=\gamma$. A simple diagram chase yields $\phi_{1} \circ\left(\psi^{\prime} \mid B\right)=\phi_{1} \circ \psi$ and hence $\psi^{\prime} \uparrow B=\psi$ as $\phi_{1}$ is onto. 
5.3 TheOREM. Assume that $\operatorname{Var} \mathscr{A}$ is subregular and let $A \in \operatorname{Var} \mathscr{A}$ be subdirectly irreducible with nonabelian monolith. Then the following are equivalent:

(i) $A$ is weakly injective in $\operatorname{Var} \mathscr{A}$;

(ii) $A$ is weakly injective relative to $\mathbf{P}(\mathscr{A})$;

(iii) $A$ is weakly injective to $\mathbf{P}_{U}(\mathscr{A})$.

Proof. (i) $\Rightarrow$ (iii) is trivial. Since $\operatorname{Var} \mathscr{A}=\operatorname{HSP}(\mathscr{A})$, (ii) $\Rightarrow$ (i) follows from the lemma above. Since ann $\mu=0$ a simple application of the Generalized Jónsson Lemma establishes (iii) $\Rightarrow$ (ii).

5.4 COROLlaRy. Let $\mathscr{A}$ be a finite set of finite algebras and assume that Var $\mathscr{A}$ is subregular. A subdirectly irreducible algebra in $\operatorname{Var} \mathscr{A}$ which has nonabelian monolith and is weakly injective relative to $\mathscr{A}$ is weakly injective in $\operatorname{Var} \mathscr{A}$.

Since a finite $A$ is trivially weakly injective with respect to $\mathscr{A}=\{A\}$, Theorem 1.2 follows at once from Corollary 5.4 .

A finite algebra $A$ is functionally complete if for all $n$ every map from $A^{n}$ to $A$ is a polynomial function (that is, a term function with some variables replaced by elements of $A$ ). A functionally complete algebra $A$ satisfies Con $A^{n} \cong 2^{n}$ for all $n$ and so is simple and, in a modular variety, nonabelian by 2.4 . It is proved in $\mathrm{H}$. Werner [15] that in a congruence permutable variety a finite algebra $A$ is functionally complete precisely when Con $A^{2} \cong 2^{2}$, that is, when $A$ is simple and nonabelian. Hence a finite group functionally complete if and only if it is simple and nonabelian and a finite ring is functionally complete if and only if it is simple and not a zero ring.

The next corollary is a partial answer to the question raised in B. A. Davey and $\mathrm{H}$. Werner [1], which motivated this work.

5.5 Corollary. Assume that $\operatorname{Var}(A)$ is subregular. If $A$ is finite, simple and nonabelian, in particular if $A$ is functionally complete, then $A$ is weakly injective in $\operatorname{Var} \boldsymbol{A}$.

Combining Corollary 5.5 with Theorem 1.8 of [1] yields our Theorem 1.3.

We now wish to show that the results in 5.1 and 5.4 for finitely generated varieties can be extended to locally finite varieties. The following result requires neither subregularity nor congruence modularity. The operator $\mathbf{P}_{f}$ denotes products of finitely many algebras.

5.6 Lemma. Assume that $\operatorname{Var} \mathscr{A}$ is locally finite and $A \in \operatorname{Var} \mathscr{A}$ is finite. If $A$ is weakly injective in the subvariety $\operatorname{Var} A$ and is weakly injective relative to $\mathbf{P}_{f}(\mathscr{A})$, then $A$ is weakly injective relative to $\mathbf{P}(\mathscr{A})$.

Proof. Assume that $A$ is weakly injective in $\operatorname{Var} A$ but is not weakly injective relative to $\mathbf{P}(\mathscr{A})$. Thus there is a subfamily $\left\{A_{i} \mid i \in I\right\}$ of $\mathscr{A}$, a subalgebra $B$ of $\Pi\left(A_{i} \mid i \in I\right)$ and a homomorphism $\phi: B \rightarrow A$ which does not extend to $\Pi\left(A_{i} \mid i \in I\right)$. Let $\psi: \Pi\left(A_{i} \mid i \in I\right) \rightarrow C$ be the largest quotient of $\Pi\left(A_{i} \mid i \in I\right)$ in $\operatorname{Var} A$. Then the 
diagram below cannot be completed by a homomorphism from $C$ to $A$.

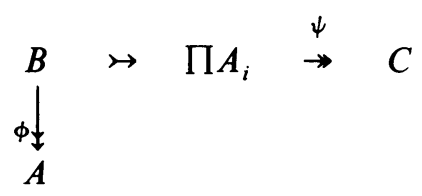

If $\operatorname{ker}(\psi \mid B) \leqslant \operatorname{ker} \phi$, then there exists a homomorphism

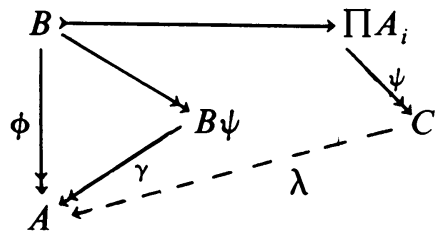

$\gamma: B \psi \rightarrow A$ such that $(\psi \uparrow B) \circ \gamma=\phi$. Since $C \in \operatorname{Var} A$ and $A$ is assumed to be weakly injective in $\operatorname{Var} A$, there exists $\lambda: C \rightarrow A$ which extends $\gamma$ and consequently $\psi \circ \lambda$ extends $\phi$, a contradiction. Thus $\operatorname{ker}(\psi \uparrow B) \nless \operatorname{ker} \phi$.

Since $B$ lies in the locally finite $\operatorname{Var} A$, some finite subalgebra $B^{\prime}$ of $B$ must have elements $x, y$ with $x \psi=y \psi$ but $x \phi \neq y \phi$, and as $A$ is finite such a $B^{\prime}$ may be chosen so that $B^{\prime} \phi=A$. Because $B^{\prime}$ is finite, $I$ must have a finite subset $J$ such that $a \uparrow J=b \uparrow J$ implies $a=b$ whenever $a, b \in B^{\prime}$. Let

$$
\tau: \prod\left(A_{i} \mid i \in I\right) \rightarrow \prod\left(A_{j} \mid j \in J\right)
$$

be the natural projection; then $\tau \uparrow B^{\prime}: B^{\prime} \rightarrow \prod A_{j}$ is an embedding.

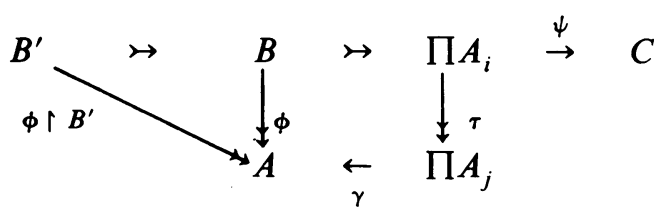

Let $\gamma: \prod A_{j} \rightarrow A$ be any homomorphism; then $\operatorname{ker} \psi \leqslant \operatorname{ker}(\tau \circ \gamma)(\operatorname{as} \operatorname{Im}(\tau \circ \gamma) \leqslant A$ $\in \operatorname{Var} A)$ and thus $x(\tau \circ \gamma)=y(\tau \circ \gamma)$. Since $x \phi \neq y \phi$ it follows that $\gamma$ does not extend $\phi \backslash B^{\prime}$. Hence $A$ is not weakly injective relative to $\mathbf{P}_{f}(\mathscr{A})$.

5.7 TheOREM. Assume that $\operatorname{Var} \mathscr{A}$ is subregular and locally finite. A finite subdirectly irreducible algebra in $\operatorname{Var} \mathscr{A}$ which has nonabelian monolith and is weakly injective relative to $\mathscr{A}$ is weakly injective in $\operatorname{Var} \mathscr{A}$.

Proof. Let $A$ be a finite subdirectly irreducible algebra with nonabelian monolith which is weakly injective relative to $\mathscr{A}$. By Theorem $1.2, A$ is weakly injective in $\operatorname{Var} A$. By Theorem 5.3, $A$ is weakly injective in $\operatorname{Var} \mathscr{A}$ precisely when it is weakly injective relative to $\mathscr{P}(\mathscr{A})$ and hence, by Proposition 5.6 above, it suffices to prove that $A$ is weakly injective relative to $\mathbf{P}_{f}(\mathscr{A})$. Since $A$ is weakly injective relative to $\mathscr{A}$, an easy application of the Generalized Jónsson Lemma shows that $\mathscr{A}$ is weakly injective relative to $\mathbf{P}_{f}(\mathscr{A})$.

It is no surprise that the local finiteness of $\operatorname{Var} \mathscr{A}$ cannot be omitted from 5.7: the set $\mathscr{A}$ of all finite nilpotent groups generates the variety of all groups, $A_{5}$ is obviously weakly injective relative to $\mathscr{A}$, yet $S_{5}$ is a proper essential extension of it. 
Under the assumptions of 5.7 it can happen that $\mathscr{A}$ consists of finite algebras yet $\operatorname{Var} \mathscr{A}$ contains infinite subdirectly irreducible algebras $A$ whose monoliths are nonabelian. Such an $A$ is trivially weakly injective relative to $\mathscr{A}$, but need not even be an absolute subretract in $\operatorname{Var} \mathscr{A}$. For example, let $p$ be a prime, $G$ a nonabelian finite simple group, $E$ any infinite elementary abelian $p$-group, and $\mathscr{A}$ the set of all finite subgroups of the standard restricted wreath product $A=G \mathrm{wr} E$. Such an $A$ is always subdirectly irreducible, the monolith being the commutator subgroup. If $E_{1}$ is a proper subgroup of $E$, then $G$ wr $E_{1}$ is a proper subgroup of $E$, isomorphic to $A$ if $E$ and $E_{1}$ have the same cardinality. Thus $A$ may be viewed as 'a proper essential extension of itself'. On the other hand, $A \in \operatorname{Var} \mathscr{A}$ and $\operatorname{Var} \mathscr{A}$ is locally finite. (One may note further that the isomorphism types of the members of $\mathscr{A}$ do not depend upon the cardinality of the infinite $E$ : thus the same $\operatorname{Var} \mathscr{A}$ has such $A$ with arbitrarily large cardinalities.)

In view of 5.7, it may be of interest to note the following:

5.8 Lemma. Assume that Var $\mathscr{A}$ is congruence modular and locally finite. If $A \in \operatorname{Var} \mathscr{A}$ is finite and subdirectly irreducible with nonabelian monolith, then $A \in$ $\mathscr{H} \mathscr{S}(\mathscr{A})$. If $A$ is also weakly injective relative to $\mathscr{A}$, then $A \in \mathbf{H}(\mathscr{A})$.

Proof. Let $|A|=n$. Since the free algebra in $\operatorname{Var} \mathscr{A}$ on $n$ generators is finite, it belongs to $\mathbf{S P}_{f}(\mathscr{A})$ and hence $A \in \mathbf{H S P}_{f}(\mathscr{A})$. Since $A$ is subdirectly irreducible with nonabelian monolith, we have ann $\mu=0$ and the Generalized Jónsson Lemma gives $A \in \mathbf{H S}(\mathscr{A})$.

6. An example. We describe the construction of a finite monolithic group $G$ in which the centralizer of the monolith is abelian yet $G$ is not an absolute subretract in the variety it generates. As acknowledged in the Introduction, this example was made in 1964 by the second author in collaboration with M. F. Newman. The verification of the relevant properties of $G$ will only be sketched here.

Let $U$ be a 6-dimensional vectorspace over the field $\mathbf{F}_{2}$ of two elements, with a basis permuted transitively by the alternating group $A_{5}$ (so the stabilizer in $A_{5}$ of a basis element is a dihedral group of order 10). Regard $U$ as an $\mathbf{F}_{2} A_{5}$-module. The

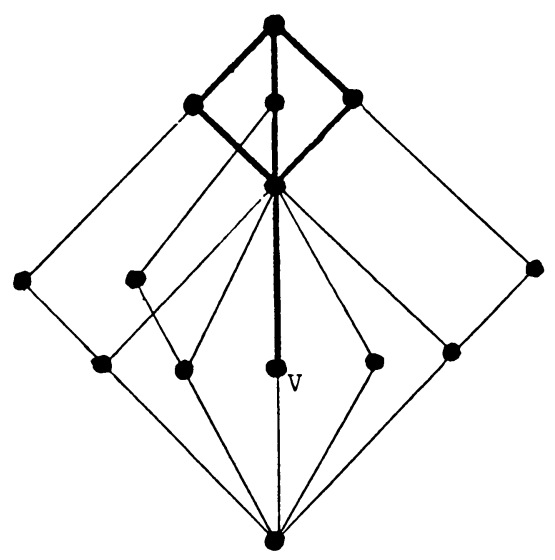

FIGURE 1 


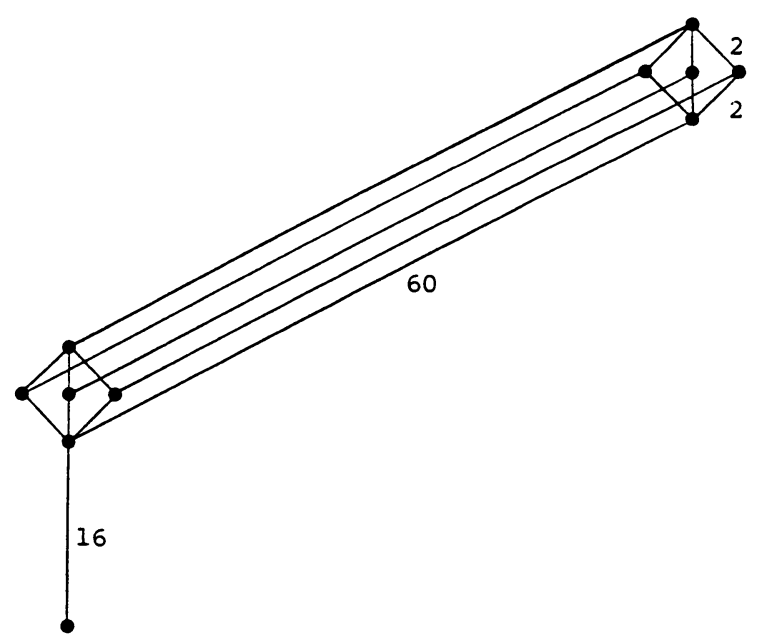

FIGURE 2

sum of the basis elements spans a 1-dimensional submodule, call that $U_{1}$; the set of the vectors whose coordinates (relative to this basis) sum to 0 , is a submodule of codimension 1: call that $U_{5}$. The only proper, nonzero submodules of $U$ are $U_{1}$ and $U_{5}$. The 1-dimensional composition factors $U_{1}$ and $U / U_{1}$ are of course absolutely irreducible, but $U_{5} / U_{1}$ is not: while $\operatorname{End} U_{1}=\operatorname{End} U / U_{5}=\mathbf{F}_{2}$, the endomorphism ring End $U_{5} / U_{1}$ is the field $\mathbf{F}_{4}$ of order 4 . It follows that End $U / U_{1}$ is also $\mathbf{F}_{2}$. Consequently, the submodule lattice of $\left(U / U_{1}\right) \oplus\left(U / U_{1}\right)$ is as shown on Figure 1.

In particular we see that there exists an $\mathbf{F}_{2} A_{5}$-module, namely the quotient modulo $V$, which is a monolithic sum of two copies of $U / U_{1}$.

Let $G$ be the semidirect product of $A_{5}$ and $U / U_{1}$. As the simple group $A_{5}$ acts faithfully on $U_{5} / U_{1}$, the only minimal normal subgroup of $G$ is $U_{5} / U_{1}$, and its centralizer is the abelian $U / U_{1}$.

To see that $G$ is not an absolute subretract in the variety it generates, consider the direct square of $G$; within this, let $S$ be the product of the diagonal copy of $G$ and the direct square of $U / U_{1}$. The $\mathbf{F}_{2} A_{5}$-submodules of the direct square of $U / U_{1}$ are normal subgroups of $S$. It is easy to see that $S / V$ is monolithic and has three (normal) subgroups of index 2 isomorphic to $G$ : so $S / V$ is a proper essential extension of $G$. This completes the sketch of the proof; Figure 2 shows the normal subgroup lattice of $S / V$, the numbers given being the relevant indices.

\section{REFERENCES}

1. B. A. Davey and H. Werner, Injectivity and Boolean powers, Math. Z. 166 (1979), 205-223.

2. B. A. Davey, K. R. Miles and V. J. Schumann, Quasi-identities, Mal'cev conditions and congruence regularity, preprint, La Trobe Univ., 1986.

3. R. Freese, On Jónsson's theorem, Algebra Univ. 18 (1984), 70-76.

4. R. Freese and R. McKenzie, Residually small varieties with modular congruence lattices, Trans. Amer. Math. Soc. 264 (1981), 419-430.

5. The commutator, an overview, preprint, Univ. of Hawaii, 1979.

6. O. C. Garcia and F. Larrión, Injectivity in varieties of groups, Algebra Univ. 14 (1982), 280-286.

7. G. Grätzer and H. Lakser, The structure of pseudocomplemented distributive lattices. III. Injectives and absolute subretracts, Tran. Amer. Math. Soc. 169 (1972), 475-487. 
8. H. P. Gumm, An easy way to the commutator in modular varieties, Arch. der Math. 34 (1980), $220-228$.

9. __ Geometrical methods in congruence modular varieties, Mem. Amer. Math. Soc. 45 No. 286 (1983).

10. J. Hagemann and C. Herrmann, A concrete ideal multiplication for algebraic systems and its relation to congruence distributivity, Arch. Math. 32 (1979), 234-245.

11. C. Herrmann, Affine algebras in congruence modular varieties, Acta Sci. Math. (Szeged) 41 (1979), 119-125.

12. L. G. Kovács and M. F. Newman, Injectives in varieties of groups, Algebra Univ. 14 (1982), $398-400$.

13. H. Neumann, Varieties of groups, Ergebnisse Math. Grenzgeb., Band 37, Springer-Verlag, Berlin, Heidelberg and New York, 1967.

14. W. Taylor, Some applications of the term condition, Algebra Univ. 14 (1982), 11-24.

15. H. Werner, Congruences on products of algebras and functionally complete algebras, Algebra Univ. 4 (1974), 99-105.

Department of Mathematics, La Trobe University, Bundoora, Victoria, 3083, Australia

Department of Mathematics, Australian National University, Canberra, ACT, 2601, Australia 